\title{
TOI, TOI, TOI! Eine Theaterwerkstatt, um Deutsch zu lernen
}

\author{
Vorgehensweise und Beispiele
}

\section{Leticia García Brea}

\begin{abstract}
„Toi, toi, toi“ ist eine Theaterwerkstatt, die am Sprachzentrum der Universität León (Spanien) angeboten wird. Ihr Ziel besteht darin, die deutsche Sprache mithilfe des Theaters auf kreative Weise $\mathrm{zu}$ erlernen und $\mathrm{zu}$ verbessern. Im Folgenden wird die Vorgehensweise der Werkstatt mit konkreten Beispielen aus dem Unterricht präsentiert.
\end{abstract}

\section{1 „Warum?” Ziel der Theaterwerkstatt}

Ausdrucksvermögen und Kommunikationsfähigkeit werden [durch DramaAktivitäten] erweitert sowie die Sensibilisierung für sprachliche Phänomene angestrebt. Gleichzeitig führen der Spaß am Spiel und das Vergnügen an der Sprache zu höherer Sprechbereitschaft und bauen Sprechhemmungen ab. (Even 2003: 63f.)

Das Ziel dieser Werkstatt ist die Verbesserung des Ausdrucksvermögens und der Kommunikationsfähigkeit sowie der Abbau der Sprechhemmungen, die Even in ihrer Publikation beschreibt. Es wird auch an der körperlichen und der non-verbalen Kommunikation durch Drama-Aktivitäten gearbeitet, die den Teilnehmern (TN) helfen sollen, sich besser auf Deutsch auszudrücken. Selbst dann, wenn ihnen in einer bestimmten Sprechsituation einmal der konkrete Wortschatz fehlen sollte. Über den Verlauf der Werkstatt und das kommunikative Vergnügen äußert sich eine der Teilnehmerinnen beispielsweise wie folgt:

„Meine Freunde fragen mich, warum ich an so einer Theaterwerkstatt teilnehme. Ich finde, dass es klar ist: Weil mir Deutsch gefällt. Aber ich werde sehr nervös, wenn ich auf Deutsch sprechen muss. Meiner Meinung nach ist das Theater ein gutes Mittel, um die Angst zu nehmen. Nach unserem ersten Tag fühlte ich mich selbstbewusster. Ich finde, dass die Arbeit mit der Musik und der Mimik sehr positiv ist. Wir arbeiten mit dem Körper und dem Geist, um die deutsche Sprache zu lernen. Ich hoffe, dass wir in den nächsten Tagen gemeinsam unsere Sprache verbessern und weiterhin die Spiele genießen!“ (Carmen Ibañez León, Teilnehmerin der Werkstatt) 


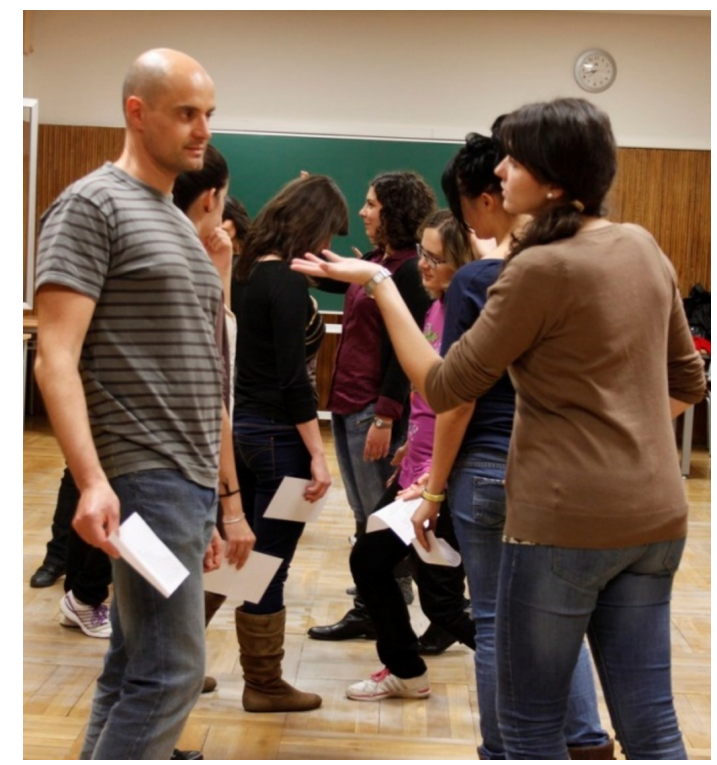

Abbildung 1: Teilnehmer an der Arbeit eines kleinen Textes mit Sprechhaltungen.

Theaterspielen bedeutet jedoch für manche Leute, lediglich einen gesprochenen Text und eine Rolle zu entwerfen und diese mit einem schönen Bühnenbild und Kostümen zu präsentieren. Etymologisch stammt das Wort Theater aus dem Altgriechischen „Theatron“, dem Raum zum Schauen. Somit ist Theater immer auch ein Kommunikationsprozess zwischen Sender (Schau-Spieler) und Empfänger (ZuSchauer). Unter Inszenierung (in Szene setzen) wird das szenische Ereignis an sich verstanden, wo die Elemente in begrenzter Zeit und an begrenztem Ort angeordnet sind, und wo jede Szene einen Kommunikationsakt darstellt. Angesichts dessen wird das Theater in dieser Werkstatt insbesondere als Kommunikationsakt verstanden. Daher wird nicht allein mit Textinszenierungen gearbeitet, sondern vor allem mit Drama-Aktivitäten. Das heißt, das Ziel besteht darin, authentische Kommunikationssituationen spielend zu erproben, sich für die echte Kommunikation auf Deutsch vorzubereiten, insbesondere durch die oben genannte Entwicklung des Ausdrucksvermögens und der Kommunikationsfähigkeit. Die TN erleben den Lernprozess, sie sind die Akteurinnen und Akteure, das heißt nicht nur passive Beobachter. Daher soll Dramapädagogik in dieser Werkstatt wie folgt verstanden werden:

Dramapädagogischer Unterricht ist damit nicht einfach nur eine Ansammlung nützlicher Theatertricks, die den Lernstoff ansprechender gestalten und die Motivation der Lernenden ankurbeln, sondern eine Unterrichtsform, die den Boden für inhaltliche, soziale und ästhetische Erkenntnisprozesse bereitet. (Even 2003: 148)

Somit wird der Lehr-Lernprozess tatsächlich handlungsorientiert: Die TN lernen durch Erfahrungen mit der Sprache im Kontext, auf der Bühne, während eines Spiels und in der Gruppe, Deutsch besser zu sprechen und den Körper bewusst in die Kommunikation einzubinden. 


\title{
2 „Wer, was, wo?” Konkretes Vorgehen
}

Die Theaterwerkstatt findet seit Oktober 2012 jeden Freitag von 17.30 Uhr bis 19.30 Uhr und von Oktober bis Mai im Sprachzentrum der Universität León statt. Die TeilnehmerInnen sind zwischen 21 und 40 Jahre alt (größtenteils Studierende oder Erwachsene mit abgeschlossenem Studium) und repräsentieren unterschiedliche Sprachniveaus (A1-B1). Sie nehmen an diesem Kurs freiwillig und kostenlos teil. Am Ende des Lehrjahres wird ein kleines Theaterstück (max. 20-25 Min.) inszeniert, in dem immer der Schaffensprozess in der Gruppe im Zentrum steht: Das heißt, die TN verfassen einen Teil des Textes und nehmen direkt an der Ausarbeitung der Inszenierung teil. Der Unterricht wird nach Oelschläger in drei Phasen unterteilt: Einstiegsphase, Erarbeitungsphase und Präsentationsphase mit Evaluation (vgl. Oelschläger 2004: 30). Die drei Phasen werden mit Beispielen genauer illustriert:

\begin{abstract}
Man beginnt mit der Vorbereitungs- bzw. Einstiegsphase, in der die Lerner auf das Thema eingestimmt werden und die Aufgabenstellung genannt wird. Darauf folgt die Erarbeitungsphase, in der in Gruppen das jeweilige Gruppenergebnis, hier also die Szene, erarbeitet wird und zuletzt die Präsentationsphase, in der die Szene dem Plenum vorgestellt wird. (ibid.)
\end{abstract}

In der Einstiegsphase handelt es sich vor allem um gezielte Aufwärmübungen, während in der Erarbeitungsphase das ausgewählte Thema vertieft und das jeweilige Gruppenergebnis erarbeitet werden. Es kann auch vorkommen, dass es in dieser Phase keine Gruppenarbeit gibt, sondern dass die TN alle zusammen und mit Hilfe der Spielleiterin (SL) im Plenum arbeiten. In der dritten Phase erfolgt die Arbeit an kleinen Szenen sowie die Improvisationen. Diese Arbeit wird in kleinen Gruppen oder Paaren organisiert und ist grundsätzlich kollaborativ. Das heißt, alle arbeiten zusammen unter Verfolgung eines gemeinsamen Zieles. Hierbei gestaltet der Lehrer den Lernprozess der TN, unterstützt sie als Lernbegleiter, womit das eigenverantwortliche Lernen in der Gruppe gefördert wird. Der Lehrer kann in dieser Phase mit genau abgestimmten Aufgaben individuell auf die verschiedenen Lerner eingehen. Diese Aufgaben können vorher sehr präzise gewählt worden sein oder spontan - je nach Bedürfnis der Lernenden - angepasst werden. Nach Abschluss der Präsentationsphase wird diese Arbeit zusammen mit den anderen Teilnehmern ausgewertet und konstruktiv evaluiert. Im Folgenden werden zwei Beispiele aus dem Unterricht dargestellt und illustriert.

\section{Direkt aus der Praxis}

„Theater macht Sprache sichtbar. Theater spricht mehrere Sprachen, es ist polyglott.“ (Andrea Breth, deutsche Theaterregisseurin) 


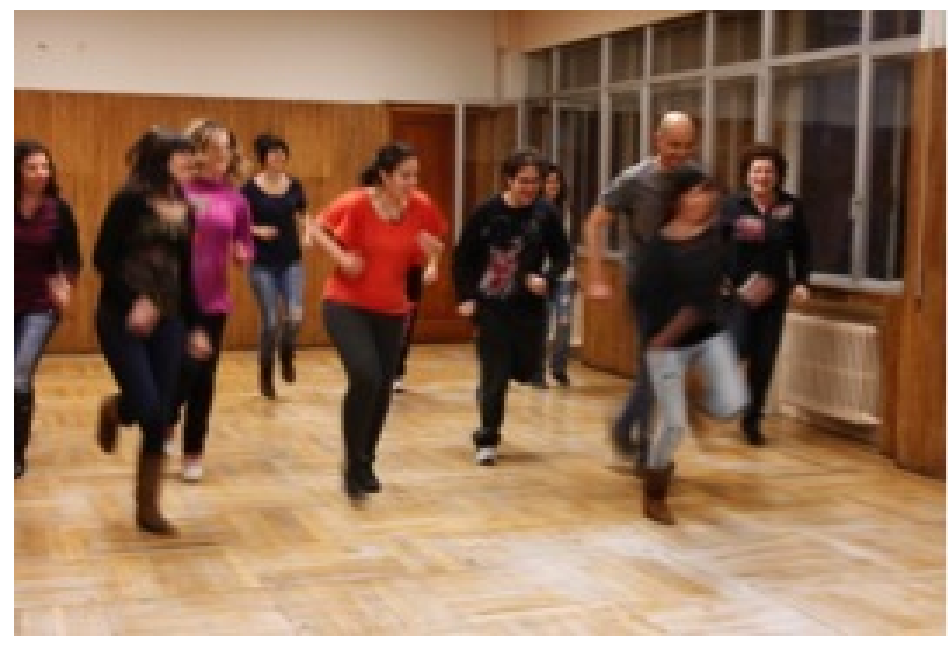

Abbildung 2: Bewegendes Lernen in der Gruppe.

\subsection{Beispiel A: Interaktives Abenteuer im Schloss}

Thema: „Atmosphäre schaffen und ein interaktives Abenteuer improvisieren.“

Einstiegsphase - Alle gehen durch den Raum und versuchen dabei, den Raum gut auszufüllen und das gleiche Tempo zu halten. Nach unterschiedlichen Aufwärmübungen gibt die SL den TN verschiedene Impulse. Verschiedene Situationen und Emotionen sollen mit dem Körper darstellt werden (z. B. es regnet, die Sonne scheint, es ist windig, wir sind auf einer Party, wir sind in einem Museum, wir sind auf einem Konzert, wir sind auf einem Begräbnis, am Strand, auf einer Demonstration etc.).

Erarbeitungsphase - Danach liest die SL aus mehreren Karteikärtchen verschiedene Situationen und Aktionen vor, die die TN darstellen sollen, z. B: „Jemand findet e i ne Tü r, ö ffnet di ese und k o mmt in e in $\mathrm{Z}$ i mmer hinein. Das Zimmer ist voller Kadaver/Ratten; mit Wasser/Blut überflutet etc.". Oder: „Jemand verwandelt sich in einen Zombie/eine Hexe/einen Vampir etc.“. In diesem Fall befinden sich die TN in einem verzauberten Schloss.

Präsentationsphase (Impro) - Vier Teilnehmer treten auf die Bühne. Sie sind in einem verzauberten Schloss und müssen verschiedene Situationen spielen. Die anderen Teilnehmer haben mehrere Kärtchen, auf denen steht, was auf der Bühne passieren soll. Der Text auf den Kärtchen wird vorgelesen. Die Spieler auf der Bühne müssen gut zuhören und die Situationen erleben sowie mit verbaler und non-verbaler Sprache spielen (sprechen).

Evaluation - Am Ende der Aktivität erfolgt die Evaluation oder Besprechung der Arbeit. Die Teilnehmer beantworten immer drei Fragen (stets konstruk- 
tive Kritik) in Bezug auf die Szenen der Mitspieler. Diese Fragen stehen normalerweise auf einem Wandplakat geschrieben:

- Was habtihrgesehen? (sachlicheBeschreibung-Anwendung des Perfekts)

- Was hat sehr gut funktioniert? (positive Aspekte hervorheben - Anwendung des Perfekts)

- Was würdet ihr verbessern? (neue Ideen hinzufügen - Anwendung des Konjunktivs)

Hier beteiligen sich alle am Gespräch. Oftmals vergessen die TN dabei beinahe, dass sie auf Deutsch sprechen. Sie wollen einfach ihre Meinung äußern, weil die Arbeit der Mitspieler sehr motivierend ist. Oftmals sprechen sie frei darüber, was sie gesehen haben. Die Sprechhemmungen werden somit signifikant reduziert. Dies ähnelt einer realen Kommunikationssituation. Somit ist unser Ziel erreicht. Die TN haben auf der Bühne das Hörverstehen und das Sprechen geübt und mit den fünf Sinnen eine interessante Szene präsentiert. Gleichzeitig mussten die anderen TN eine Geschichte mit den Karteikärtchen selbst organisieren und kreieren, sozusagen als "game masters" eines Rollenspieles. Sie wollten die Erfahrung wiederholen, weil dieser Unterricht sehr erfolgreich und motivierend war. $^{1}$

\subsection{Beispiel B: Der Tratsch}

Wer hat nicht schon einmal über andere Personen getratscht? Haben Sie nie jemandem etwas erzählt, das Sie eigentlich nicht hätten sagen dürfen? Genau darum geht es in dieser Unterrichtsstunde. Das bedeutet nicht, dass die TN Klatschmäuler sind. Vielmehr handelt es sich um eine abwechslungsreiche Übung, die darauf abzielt, die Alltagssprache anzuwenden.

Einstiegsphase - Alle gehen durch den Raum und versuchen dabei, den Raum gut auszufüllen und das gleiche Tempo zu halten. Nach verschiedenen Aufwärmübungen spielen die TN das Spiel „Ich-kann-sehr-gut“. Alle stehen in einem Kreis, jeder TN sagt, was er/sie toll/sehr gut/gut/nicht gut/überhaupt nicht machen kann. Alle Spieler, die übereinstimmen, springen auf, rufen „Ich auch/ich auch nicht!“ und tauschen ihre Plätze untereinander im Kreis. Die anderen Spieler schweigen und bleiben stehen. Der Wortschatz von Freizeit, Tagesablauf und Hobbys wird aktiviert, schwierige Strukturen für Anfänger werden eingeübt.

\footnotetext{
${ }^{1}$ Video der Szene auf Youtube: https://www youtube.com/watch?v=5SIBV7rCVs 8 (Accessed 29 September 2014).
} 
Erarbeitungsphase -

Die Teilnehmer sitzen im Kreis. TN1 erzählt dem Nachbarn/der Nachbarin z. B. Folgendes:

TN1: Ïch kann sehr gut singen, aber ich kann überhaupt nicht Tennis spielen. [Satzklammer mit Modalverb „können“-A1]

Die Nachbarin/der Nachbar hört gut zu und verspricht, nichts weiterzusagen.

TN2: „Oh, das ist aber schade! Mach dir keine Sorgen, ich sage nichts. Ich verspreche es dir!" [Alltagssprache, neue Ausdrücke, Chunks. Die SL schreibt einige neue Ausdrücke an die Tafel oder auf ein Plakat, erklärt sie und gibt Beispiele ihrer direkten Anwendung.]

Anschließend geht sie/er zu einem anderen TN und erzählt:

TN2: „Wusstest du schon? TN1 hat mir gesagt, dass sie/er sehr gut singen kann, aber dass sie/er überhaupt nicht Tennis spielen kann!"

Nebensätze mit Modalverben-A1/A2

Und der Nachbar/die Nachbarin reagiert:

TN3: „O Gott!! Wirklich? Wie kann das sein?"

Und es geht weiter:

TN4: „TN3 hat mir gesagt, dass TN2 ihr/ihm gesagt hat, dass TN1 überhaupt nicht Tennis spielen kann." [Nebensätze mit Modalverben und Perfekt-A2].

In einer zweiten Runde können die Teilnehmer immer etwas hinzufügen: TN4: „TN3 hat mir gesagt, dass TN2 ihr/ihm gesagt hat, dass TN1 überhaupt nicht Musik spielen kann und auch, dass sie nicht tanzen kann."

Nach dieser Arbeit teilen sich die TN in kleine Gruppen und bereiten kurze Szene vor, in denen sich die vorherigen Situationen entwickeln sollen oder in denen zumindest mit der schon erarbeitete Struktur gespielt wird.

Präsentationsphase — Die Szenen werden präsentiert und beobachtet. Evaluation:

- Washabtihrgesehen? (sachlicheBeschreibung-Anwendung des Perfekts)

- Was hat sehr gut funktioniert? (positive Aspekte hervorheben - Anwendung des Perfekts)

- Was würdet ihr verbessern? (neue Ideen hinzufügen - Anwendung des Konjunktivs)

Alles sollte natürlich sehr übertrieben und theatralisch gespielt werden. Die Teilnehmer lachen und sprechen sehr viel. Außerdem üben sie die Anwendung der Satzklammer und eine weitere schwierige Struktur, nämlich die der Nebensätze mit „dass“. Durch das gemeinsame Lernen und Üben steigt die Motivation. 


\subsection{Werkschau am Ende des Lehrjahres}

Am Ende des ersten Lehrjahres kam plötzlich die Frage: „Werden wir etwas inszenieren?" Das Ziel des Theaterkurses war nicht, ein Theaterstück vorzubereiten, um es vor Publikum zu inszenieren. Doch die Teilnehmer wollten von sich aus etwas vorführen. So ist zum Beispiel auch eine Werkschau ein konkretes Lernziel: Es bietet den Anreiz, sich in bewusster Form zu präsentieren und fördert die Motivation an der Fremdsprache. ${ }^{2}$ Also haben wir einzelne Ideen aus dem Unterricht genommen. Hieraus ist ein kleines Stück (20-25 Min.) entstanden. Wir haben „Vater \& Sohn Bildergeschichten“ (e. o. plauen) ausgewählt und diese inszeniert. Die TN haben den deutschen Text selbst geschrieben und die Geschichte mittels eines kreativen Prozesses erschaffen, an dem wir alle (Lehrerin und TN) teilgenommen haben.

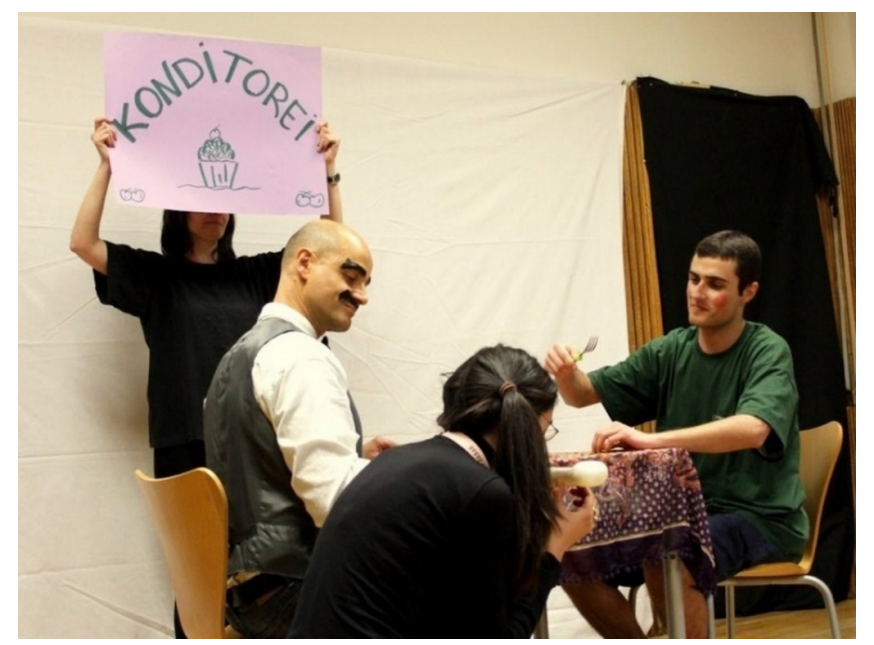

Abbildung 3: Szene in der Konditorei von Vater \& Sohn

Der Gestaltungsprozess folgte dabei dem Prinzip: Vom Bild zum Standbild, vom Standbild zur Bewegung, von der Bewegung zum Text. Dadurch wurde die non-verbale Sprache gefördert und die Dialoge entstanden aus den Situationen heraus. Da der Text auch von den Teilnehmern geschrieben wurde, haben sie ihn in bewusster Form gelernt und dargestellt. Dies ging einher mit einer motivierenden Vertiefung in den Bereichen Phonetik, Satzmelodie und Intonation. Das Video zum „Inszenierungsprozess von Vater\&Sohn“ findet sich unter: https://www.youtube.com/watch?v=KLGhZTYhyTc (Accessed 29 September 2014).

Am Ende des zweiten Lehrjahres (Mai 2014) hat die Gruppe etwas Neues ausprobiert: Die Bremer Stadtmusikanten \& Die spanischen Stadtmusikanten 2.0. Die TN haben das berühmte Märchen der Gebrüder Grimm inszeniert und auch eine sehr aktuelle Version des Märchens kreiert, die die gegenwärtige Situation in Spanien in Bezug auf Deutschland darstellen sollte - alles

\footnotetext{
${ }^{2}$ Vgl. hierzu auch Hensels Beitrag „Der künstlerisch-ästhetische Aspekt im dramapädagogischen Fremdsprachenlernen: Ein Kurskonzept“ in dieser SCENARIO-Sonderausgabe.
} 
selbstverständlich aus einer humorvollen Perspektive. In diesem Fall hatte das Theaterstück zwei Teile. Im ersten Teil haben die TN mit einem Text, der schon geschrieben war (ein kleines Kindertheaterstück über die Bremer Stadtmusikanten), gearbeitet. Im zweiten Teil haben sie - basierend auf diesem Text und auf derselben Struktur - eine neue und modernere Version mithilfe der SL geschrieben. Durch diese Arbeit konnte die deutsche Literatur auf abwechslungsreiche Weise in den kreativen Prozess eingebunden werden.

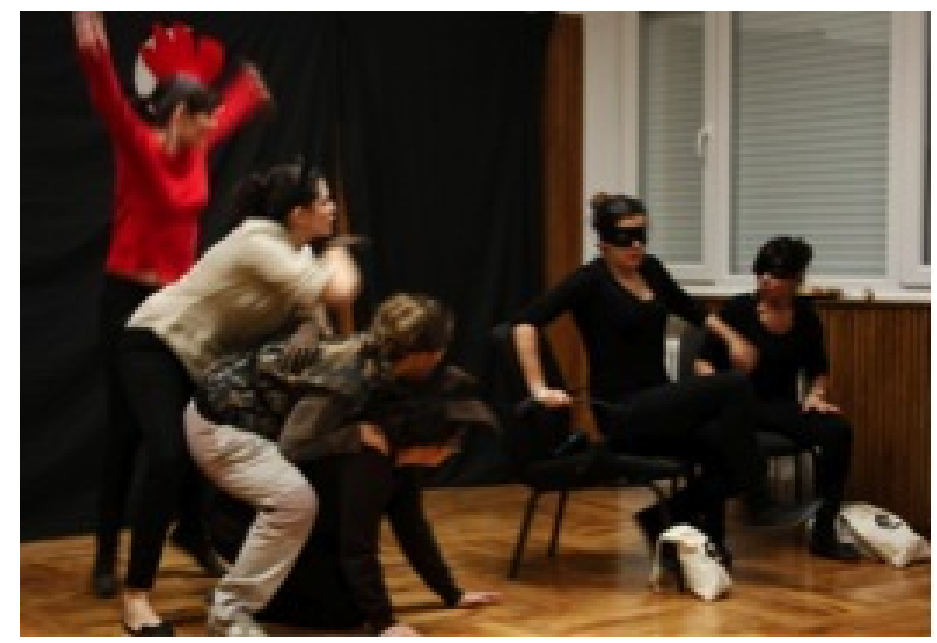

Abbildung 4: Beispiel einer Szene aus der Inszenierung der Bremer Stadtmusikanten.

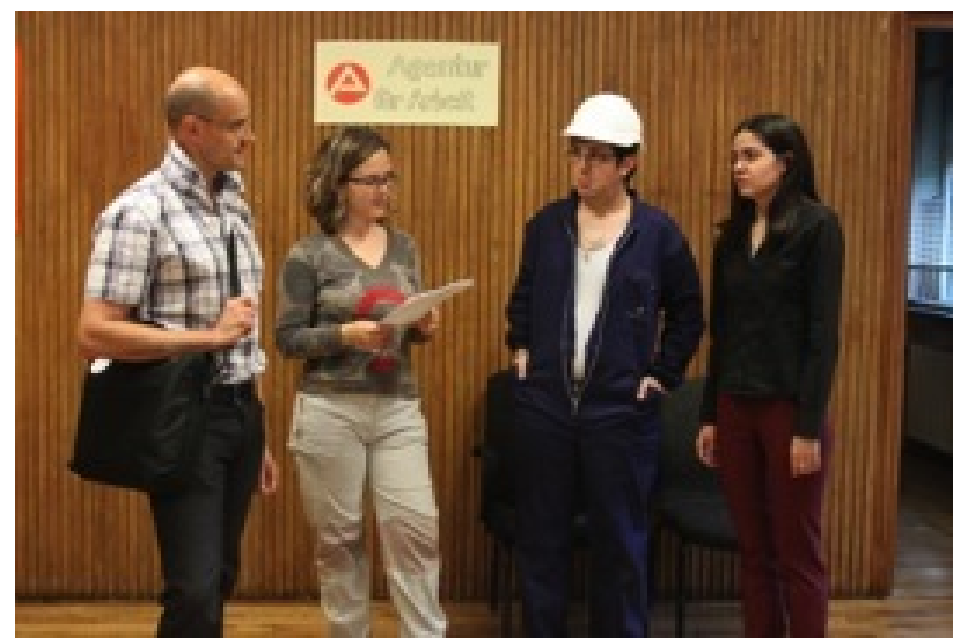

Abbildung 5: Weiteres Beispiel einer Szene aus der Inszenierung der Bremer Stadtmusikanten.

Ziel der Werkstatt ist es also, einen dramapädagogischen Unterricht durchzuführen, in dem das Lernen als Prozess und Erfahrung verstanden wird. Der Prozess ist das Lernziel - und nicht das Theaterstück an sich. Auch die Werkschau selbst ist ein Teil des Prozesses, und nicht nur das Endprodukt der 
Theaterwerkstatt. Es ist für die TN eine ganzheitliche Erfahrung.

Ich kann jeden leeren Raum nehmen und ihn eine Bühne nennen. Ein Mann geht durch den Raum, während ihm ein anderer zusieht; das ist alles, was zur Theaterhandlung notwendig ist. (Brook 1985: 9)

In beiden Werkschauen hatten wir dasselbe Problem: Wir wollten vor einem Publikum spielen, aber in unserer Stadt (León) gibt es kein großes Publikum, das Deutsch versteht. Deswegen mussten wir ein Stück vorbereiten, das eine universelle Sprache benutzen würde - das heißt: Mimik. Die Bildgeschichten von e. o. plauen waren hierfür ideal, da sie nur die zeichnerische Sprache gebrauchen und keine Wörter benötigen, um Geschichten und Witze zu erzählen. Die Umsetzung fiel insofern leichter, als Gestik und Mimik die Zeichnungen darstellen sollten und die Wörter nicht so viel Gewicht hatten. In der zweiten Werkschau haben wir eine weltbekannte Geschichte ausgewählt, die genau auf unsere gegenwärtige Situation - nämlich der wirtschaftlichen und sozialen Krise in Spanien - passte. Wir haben die zwei Situationen verglichen: Die Bremer Stadtmusikanten haben den Wunsch, nach Bremen zu reisen, um ein gutes Leben zu haben. In „Die spanischen Stadtmusikanten“ wird über die Migrationswelle von spanischen Jugendlichen gesprochen, die nach Deutschland auswandern, um eine Arbeit und ein besseres Leben zu finden. Im Vordergrund beider Geschichten steht die Moral: Das gemeinsame Verfolgen eines Zieles führt zum Erfolg.

\section{Und „Wozu?" als Schlusswort}

Innerhalb der Gruppe, die zusammen Theater spielt, entsteht ein Gefühl der Gemeinsamkeit sowie eine stressfreie Atmosphäre, in der man sich wohlfühlt. Das Selbstwertgefühl und die Empathiefähigkeit nehmen zu. Die größten Sprechhemmungen werden somit einfacher abgebaut, da alle Drama-Aktivitäten und Improvisationen mit hoher Intensität gespielt werden. Dramapädagogik ist ein bereicherndes Instrument, um die konkreten Lehr-Lernprozesse im Deutschals-Fremdsprache-Unterricht zu vertiefen. Sie macht die TN handlungsfähiger und schafft ein detailliertes Verständnis bzw. eine bewusstere Wahrnehmung der Sprache und ihrer Gesten. Hierzu ein exemplarischer Eindruck:

„Theater auf Deutsch zu spielen, ist eine gute Idee gewesen. Ich habe viele neue Wörter gelernt und vor allem haben wir unsere Aussprache verbessert. Das heißt, wir haben gelernt, flüssiger zu s p rechen. Außerdem haben wir gelernt, wie man auf Spanisch „den Chip geändert“ (umdenken) sagt. Großartig war auch die tolle Stimmung in der Gruppe! In der letzten Zeit habe ich auch bemerkt, dass mir Theater richtig gefällt. Und nicht nur, weil ich Deutsch lerne, sondern wegen der Darstellung, des Auftretens. Ich freue mich auf unsere Werkschau in diesem Jahr und habe auch viel Lust, nächstes Jahr weiterzumachen!" (Luis Enrique Martínez Palomo, Teilnehmer der Werkstatt) 
Diese und weitere Kommentare kann man auf dem Blog unserer Theaterwerkstatt lesen: http://toitoitoitheater.blogspot.com.es (Accessed 29 September 2014). Zu finden sind dort auch Videos, Fotos und Praxisberichte über weitere Theateraktivitäten.

\section{Bibliografie}

Brook, Peter (1985): Der leere Raum. Berlin: Alexander Verlag

Even, Susanne (2003): Drama Grammatik. Dramapädagogische Ansätze für den Grammatikunterricht Deutsch als Fremdsprache. München: Iudicium Verlag

Oelschläger, Birgit (2004): Szenisches Spiel im Unterricht „Deutsch als Fremdsprache“. In: GFL Journal, 1-2004, 24-34. Online:

http://www.gfl-journal.de/1-2004/oelschlaeger.pdf (Accessed 29 September 2014)

http://toitoitoitheater.blogspot.com.es (Accessed 29 September 2014) https://www • youtube.com/watch?v=KLGhZTYhyTc (Accessed 29 September 2014)

https://www youtube. com/watch?v=5SIBV7rCVs8 (Accessed 29 September 2014)

Links - 